\title{
Extended Esophagectomy in Elderly Patients with Esophageal Cancer: Minor Effect of Age Alone in Determining the Postoperative Course and Survival
}

\author{
B. B. Pultrum, MD, PhD ${ }^{1}$, D. J. Bosch, BSc ${ }^{1}$, M. W. N. Nijsten, MD, $\mathrm{PhD}^{2}$, M. G. G. Rodgers, $\mathrm{MD}^{2}$, H. Groen, MD, \\ $\mathrm{PhD}^{3}$, J. P. J. Slaets, MD, $\mathrm{PhD}^{4}$, and J. Th. M. Plukker, MD, PhD ${ }^{1}$ \\ ${ }^{1}$ Department of Surgery/Surgical Oncology, University Medical Center Groningen, University of Groningen, Groningen, \\ The Netherlands; ${ }^{2}$ Surgical Intensive Care Unit, University Medical Center Groningen, University of Groningen, \\ Groningen, The Netherlands; ${ }^{3}$ Department of Epidemiology, University Medical Center Groningen, University of \\ Groningen, Groningen, The Netherlands; ${ }^{4}$ Department of Geriatrics, University Medical Center Groningen, University \\ of Groningen, Groningen, The Netherlands
}

\begin{abstract}
Background. Elderly patients who undergo esophagectomy for cancer often have a high prevalence of coexisting diseases, which may adversely affect their postoperative course. We determined the relationship of advanced age (i.e., $\geq 70$ years) with outcome and evaluated age as a selection criterion for surgery.

Methods. Between January 1991 and January 2007, we performed a curative-intent extended transthoracic esophagectomy in 234 patients with cancer of the esophagus. Patients were divided into two age groups: $<70$ years (group I; 170 patients) and $\geq 70$ years (group II; 64 patients).

Results. Both groups were comparable regarding comorbidity (American Society of Anesthesiologists classification), and tumor and surgical characteristics. The overall in-hospital mortality rate was $6.2 \%$ (group I, $5 \%$, vs. group II, $11 \%, P=0.09$ ). Advanced age was not a prognostic factor for developing postoperative complications (odds ratio, 1.578; 95\% confidence interval, 0.857-2.904; $P=0.143)$. The overall number of complications was equal with $58 \%$ in group I vs. $69 \%$ in group II $(P=0.142)$.
\end{abstract}

Presented at the Gastrointestinal Cancers Symposium 2009 in San Francisco, CA, and at the 62nd Cancer Symposium 2009 of the Society of Surgical Oncology in Phoenix, AZ.

(C) The Author(s) 2010. This article is published with open access at Springerlink.com

First Received: 13 May 2009;

Published Online: 24 February 2010

B. B. Pultrum, MD, $\mathrm{PhD}$

e-mail: b.b.pultrum@chir.umcg.nl
Moreover, the occurrence of complications in elderly patients did not influence survival $(P=0.174)$. Recurrences developed more in patients $<70$ years $(58 \%$ vs. $42 \%, P=0.028$ ). The overall 5 -year survival was $35 \%$, and, when included, postoperative mortality was $33 \%$ in both groups $(P=0.676)$.The presence of comorbidity was an independent prognostic factor for survival $(P=0.002)$. Conclusions. Advanced age ( $\geq 70$ years) has minor influence on postoperative course, recurrent disease, and survival in patients who underwent an extended esophagectomy. Age alone is not a prognostic indicator for survival. We propose that a radical resection should not be withheld in elderly patients with limited frailty and comorbidity.

Esophageal cancer predominantly occurs in the last decades of life, with a median age of $>60$ years. ${ }^{1,2}$ Although important improvements have been achieved in the multimodality treatment of these tumors, surgery remains the primary curative option. ${ }^{3,4}$ Esophagectomy is a high-risk procedure with serious postoperative complications and a reported mortality rate ranging from 2 to $6 \% .^{1,3,5}$ Moreover, esophageal cancer patients often have considerable risk factors for major surgery, including obesity and pulmonary and cardiovascular diseases. ${ }^{6,7}$

Besides the increasing incidence of esophageal adenocarcinoma, the rising life expectancy in the general population is responsible for a relatively large number of elderly patients with esophageal cancer. ${ }^{1}$ Elderly patients who undergo esophagectomy often have a high prevalence of comorbidity and frailty, suggesting a negative effect on 
the outcome and postoperative course.$^{8-12}$ Therefore, surgeons are in general more reluctant to perform major surgery in these elderly patients.

There is a lack of evidence regarding the appropriate surgical treatment of esophageal cancer in the elderly population. Some authors propose a transhiatal procedure for better short-term outcome with less morbidity, while others perform a standard extended esophagectomy with a two-field lymphadenectomy in all patients with esophageal carcinoma to achieve maximal oncological control and minimizing the chance of recurrent disease. $^{13-15}$

In the absence of an established definition of elderly patients regarding high-risk surgery, most studies defined advanced age as $\geq 70$ years. $8,10,11,16-22$

We report the results from an experienced high-volume single center in elderly patients who underwent extended transthoracic esophagectomy with two-field lymphadenectomy for cancer of the esophagus. We performed several analyses to determine the effect of advanced age on comorbidity, postoperative course, recurrent disease, and survival. We evaluated age as a selection criterion for surgery and make recommendations for the optimal treatment policy in elderly patients.

\section{PATIENTS AND METHODS}

\section{Patient Characteristics and Treatment}

Between January 1991 and January 2007, a total of 234 patients with cancer of the esophagus or gastroesophageal junction underwent esophagectomy with curative intent. All patients underwent surgery in the same high-volume university medical center by the same surgical group, which consisted of two surgeons. All included patients were medically fit enough to undergo surgery. Patients who underwent neoadjuvant treatment in a nationwide trial, starting from 2006 on, were excluded for evaluation to prevent treatment bias $(n=6)$. Patients who underwent exploration for unforeseen extension of disease were excluded.

\section{Comorbidity}

Comorbidity was determined by the American Society of Anesthesiologists (ASA) physical status classification. ASA is a readily available and widely accepted way to stratify surgical patients according to their perioperative risk and varies between ASA class 1 (very good condition) and ASA class 5 (moribund patient). ${ }^{23}$ ASA class was assigned by the anesthesiologist after completing a structured review of physical status just before esophagectomy.

\section{Preoperative Workup}

Patients were considered for curative esophagectomy after a complete preoperative workup, which included physical examination, standard laboratory tests, digestive endoscopy, histopathological examination of biopsy samples, and detailed preoperative risk assessments. Staging of the tumors was performed by endoscopic ultrasound with fine-needle aspiration and computed tomography (CT) of the chest, abdomen, and cervical region. In all patients newly diagnosed with T3-4 or N1 esophageal cancer, a ${ }^{18}$ F-fluoro-2-deoxy-D-glucose positron emission tomography (PET) scan was performed. In case of anatomical difficulties on PET assessment, a PET/CT fusion scan was performed. Before surgery, all patients were discussed in multidisciplinary meetings.

\section{Surgery}

An extended esophageal resection was performed in all patients. This procedure consisted of a subtotal esophageal resection through a left thoracolaparotomy with intrathoracic anastomoses for the distal and gastroesophageal cancers or through a right thoraco-mid laparotomy with cervical anastomoses for the higher intrathoracic tumors. Both were combined with a two-field lymphadenectomy of nodes at the celiac trunk, upper border of the pancreas, para-aortic region, and mediastinal nodes.

\section{Histology}

All resected specimens and lymph nodes were examined according to standard procedures. Tumor stage and grade were classified according to the 6th edition of the tumor, node, and metastasis system and the residual tumor (R) classification of the International Union Against Cancer and the American Joint Committee on Cancer. ${ }^{24,25}$ Adenocarcinomas observed on hematoxylin and eosin-stained samples were confirmed in all cases by keratin staining (immunohistochemical analysis).

\section{Mortality and Complications}

Postoperative mortality was defined as any death within the same hospital admission and all deaths within the first 90 days after surgery. A separate calculation was made of only 90-day mortality (without in-hospital deaths after 90 days) to compare these figures with the data in literature. Major complications were divided into pulmonary complications: respiratory insufficiency (prolonged need for mechanical ventilation), acute respiratory distress syndrome (acute and persistent lung inflammation with increased vascular permeability and severe hypoxemia), 
pneumonia (infiltrate on $\mathrm{x}$-ray, sepsis, and positive sputum culture, including bronchoalveolar lavage), atelectasis (collapse of lung lobe on x-ray with hypoxemia for which intensive physiotherapy or bronchoscopy was needed), pleural effusion (fluid seen on $\mathrm{x}$-ray for which drainage was necessary because of hypoxemia), empyema (positive culture or positive fluid) and pulmonary embolism (diagnosed by CT); cardiac complications; arrhythmia (diagnosed on electrocardiogram) and myocardial infarction (diagnosed on electrocardiogram and positive laboratory tests); and other major complications; rebleeding (bleeding requiring transfusion or reoperation), subphrenic abscess and/or intra-abdominal abscess (CT, drainage, and positive culture), systemic inflammatory response syndrome (SIRS; deregulated host with inflammatory response with absent infection: temperature $>38.5$ or $<35^{\circ} \mathrm{C}$, heart rate $>90$ beats/min, respiratory rate $>20$ breaths $/ \mathrm{min}$ or $\mathrm{PaCO}_{2}<32 \mathrm{~mm} \mathrm{Hg}$ and white blood cells $>12,000$ cells $/ \mathrm{mm}^{3}$ ), sepsis (the clinical signs of SIRS, but with culture-proven infection or an infection identified by visual inspection), anastomotic leakage (CT with enteral contrast and amylase in the pleural fluid), chylothorax (chyle defined by measuring triglycerides), renal failure (increasing creatinine and oliguria for which renal replacement therapy was necessary), liver failure (increasing bilirubin, liver enzymes, lactate, and prothrombin time), and deep venous thrombosis (of the distal or proximal lower extremity) and ileus (absence of peristalsis with gastric retention and no defecation, confirmed with abdominal X-ray). Minor complications were defined as wound infections (positive wound culture with pus), wound dehiscence (spontaneous opening of the fascia) and urinary tract infections (sepsis, urinary leucocytes, and $10^{5}$ bacteria/ml in urine). Infectious complications were subdivided into septic complications (sepsis) and intraabdominal or subphrenic abscess eventually with anastomotic leakage, empyema, pneumonia, severe wound infections, and urinary tract infections. The use of antibiotics and inotropes was scored during the postoperative period. The operation room time, intensive care unit (ICU) length of stay, and hospital length of stay were measured for comparison.

\section{Follow-up}

All medical follow-up data were collected prospectively in a patient research database. Patients were seen in the outpatient department every 3 months for the first postoperative year, every 6 months for the next year, and then annually for 10 years. Data of deceased patients were collected by consulting the general practitioners and the Comprehensive Cancer Center, North Netherlands. Followup was measured in months from the time of operation until death (survival time) or end of follow-up with a minimum of 2 years.

For the calculation of long-term cancer-specific survival, patients without postoperative mortality were selected $(n=219)$, and only cancer-related cause of death was scored. Death by any other cause was scored as end of follow-up. For all other survival calculations, postoperative mortality was included in the survival curves.

Recurrent disease was defined as locoregional recurrence or distant metastases in the follow-up period, determined by any cytologic or histologic proof, unequivocal radiologic suspicion (CT, magnetic resonance imaging, PET, bone scan, and ultrasound), and/or obvious clinical manifestations.

The follow-up was ascertained in February 2009 and was complete for all included patients.

\section{Definitions and Statistical Analyses}

For calculations, "elderly" was defined as a patient $\geq 70$ years of age, as generally used in the literature. ${ }^{8,10,11,16-22}$ Therefore, we discriminated between group I, $<70$ years, and group II, $\geq 70$ years, independently of other factors.

Variables were reflected as frequencies with means and/ or median with percentages. Continuous variables were compared by the $t$-test, and the $\chi^{2}$ test was used for comparison of categorical variables. Survival and recurrence rates were calculated by the Kaplan-Meier method and the $\log$ rank test. Survival calculations included postoperative mortality, except for the cancer-specific survival. Prognostic factors for survival were calculated with Cox regression univariate and multivariate analyses. Univariate and multivariate logistic and linear regression analysis were used for calculating if advanced age was influencing the occurrence of comorbidity and complications; the group of complications (cardiac, pulmonary, and infectious complications) were calculated as well as individual complications. Multivariate analysis was performed by incorporating factors as covariates with a $P$ value of $\leq 0.1$ on univariate analysis.

For all calculations, a $P$ value of $<0.05$ was considered to be significant. Statistical computations and figuring were all performed by SPSS software, version 16.0 (SPSS, Chicago, IL).

\section{RESULTS}

\section{Patients Characteristics}

The study population consisted of 234 consecutive patients, 196 men (84\%) and 38 women (16\%). The mean 
age at operation was 63 years, with a range of 28 to 82 years. Group I ( $<70$ years of age) consisted of 170 patients $(73 \%)$ and group II ( $\geq 70$ years of age) of 64 patients $(27 \%)$. Patients and tumor characteristics are summarized in Table 1. Surgical characteristics such as year of surgery $(P=0.4)$ and type of resection $(P=0.9)$ were similar in both groups. Stage of disease was not statistically different in both groups $(P=0.148)$, although more advanced disease seemed to occur in the younger patients (Table 1).

Comorbidity was not significantly different in both groups: 65 patients $(38 \%)$ with comorbidity in group I versus 32 patients $(50 \%)$ in group II $(P=0.104)$. ASA class did not differ between the two groups $(P=0.136)$.

\section{Mortality and Comorbidity}

Postoperative mortality (defined as within 90 days of surgery and within-hospital admission) was $6.2 \%$ (15 patients), 8 patients (5\%) in group I versus 7 patients $(11 \%)$ in group II $(P=0.09)$. The 90 -day mortality alone was $4.7 \%$ (11 patients), with 5 patients $(3 \%)$ in group I and 6 patients $(9 \%)$ in group II $(P=0.08)$. Of the 15 patients who died after surgery, $10(67 \%)$ had more than one comorbidity $(P=0.041)$. Four patients had a history of myocardial infarction and hypertension; one patient had diabetes with myocardial infarction; two patients had diabetes with hypertension; one patient had chronic obstructive pulmonary disease with transient ischemic attack; and two patients had a transient ischemic attack with hypertension. Only cardiovascular comorbidity in the elderly subgroup $(n=24 ; 38 \%)$ had a negative effect on postoperative mortality $(P=0.043)$.

\section{Complications}

Ninety-nine patients (58\%) in group I and 44 patients (69\%) in group II developed postoperative complications, which was not statistically different between both groups $(P=0.142)$. Pulmonary complications occurred in 72 patients (42\%) in group I versus 36 patients $(56 \%$; $P=0.06$ ) in group II, respectively (Table 2 ). Respiratory insufficiency was the most frequent complication and occurred more in the elderly patients (25 vs. $41 \%$; $P=0.017)$. Other major pulmonary complications, atelectasis $(14 \%$ vs. $28 \% ; P=0.009)$ and pleural effusion
TABLE 1 Characteristics of elderly patients with esophageal cancer $(n=234)$ and of tumor
$G E J$ gastroesophageal junction, $C O P D$ chronic obstructive pulmonary disease, TIA/CVA transient ischemic attack/ cerebral vascular accident

\begin{tabular}{|c|c|c|c|}
\hline Variable & $\begin{array}{l}\text { Group I } \\
(\text { age }<70) \\
(N=170)\end{array}$ & $\begin{array}{l}\text { Group II } \\
(\text { age } \geq 70) \\
(N=64)\end{array}$ & $\begin{array}{l}P \\
\text { value }\end{array}$ \\
\hline Mean age $(y)$ & 58.9 & 74.5 & \\
\hline $\operatorname{Sex}(M / F)$ & $144 / 26$ & $52 / 12$ & 0.524 \\
\hline \multicolumn{4}{|l|}{ Histology, $n(\%)$} \\
\hline Adenocarcinoma & $145(85)$ & $56(87)$ & 0.666 \\
\hline $\begin{array}{l}\text { Squamous-cell } \\
\text { carcinoma }\end{array}$ & $25(15)$ & $8(13)$ & \\
\hline \multicolumn{4}{|l|}{ Localization, $n(\%)$} \\
\hline Midesophagus & $14(8)$ & $4(6)$ & 0.371 \\
\hline Distal esophagus & $102(60)$ & $36(56)$ & \\
\hline GEJ & $54(32)$ & $24(38)$ & \\
\hline \multicolumn{4}{|l|}{ Tumor stage, $n(\%)$} \\
\hline I & $17(10)$ & $11(17)$ & 0.148 \\
\hline IIa & $46(27)$ & $17(26)$ & \\
\hline $\mathrm{IIb}$ & $16(9)$ & $8(13)$ & \\
\hline III & $80(47)$ & $25(39)$ & \\
\hline IVa & $11(7)$ & $3(5)$ & \\
\hline \multicolumn{4}{|l|}{ Comorbidity, $n(\%)$} \\
\hline Diabetes mellitus & $17(10)$ & $8(13)$ & 0.582 \\
\hline Hypertension & $28(16)$ & $15(23)$ & 0.221 \\
\hline Angina pectoris & $12(7)$ & $5(8)$ & 0.843 \\
\hline Heart failure & $1(1)$ & $2(3)$ & 0.125 \\
\hline Myocardial infarction & $17(10)$ & $7(11)$ & 0.833 \\
\hline COPD & $16(9)$ & $6(9)$ & 0.993 \\
\hline TIA/CVA & $7(4)$ & $6(9)$ & 0.118 \\
\hline
\end{tabular}


TABLE 2 Complications in elderly patients with esophageal cancer $(n=234)$
ARDS acute respiratory distress syndrome, SIRS systemic inflammatory response syndrome, ICU intensive care unit

Bold values are significant $(P \leq 0.005)$

\begin{tabular}{|c|c|c|c|}
\hline Variable & $\begin{array}{l}\text { Group I (age < 70), } n(\%) \\
(N=170)\end{array}$ & $\begin{array}{l}\text { Group II (age } \geq 70), n(\%) \\
(N=64)\end{array}$ & $\begin{array}{l}P \\
\text { value }\end{array}$ \\
\hline Overall complications & $99(58)$ & $44(69)$ & 0.142 \\
\hline Pulmonary complications & $72(42)$ & $36(56)$ & 0.058 \\
\hline Respiratory insufficiency & $42(25)$ & $26(41)$ & $\mathbf{0 . 0 1 7}$ \\
\hline ARDS & $4(2)$ & $3(5)$ & 0.351 \\
\hline Pneumonia & $28(16)$ & $15(23)$ & 0.221 \\
\hline Atelectasis & $23(14)$ & $18(28)$ & 0.009 \\
\hline Pleural effusion & $25(15)$ & $17(27)$ & 0.036 \\
\hline Empyema & $16(9)$ & $9(14)$ & 0.306 \\
\hline Pulmonary embolism & $4(2)$ & $3(5)$ & 0.351 \\
\hline Cardiac complications & $27(16)$ & $24(38)$ & $<\mathbf{0 . 0 0 1}$ \\
\hline Arrhythmia & $27(16)$ & $23(36)$ & 0.001 \\
\hline Myocardial infarction & $2(1)$ & $1(2)$ & 0.815 \\
\hline \multicolumn{4}{|l|}{ Other major complications } \\
\hline Rebleeding & $4(2)$ & $4(6)$ & 0.144 \\
\hline Subphrenic abscess & $0(0)$ & $2(3)$ & 0.021 \\
\hline SIRS & $3(2)$ & $4(6)$ & 0.073 \\
\hline Sepsis & $14(8)$ & $9(14)$ & 0.183 \\
\hline Anastomotic leakage & $31(18)$ & $8(13)$ & 0.295 \\
\hline Chylothorax & $9(5)$ & $2(3)$ & 0.486 \\
\hline Intra-abdominal abscess & $0(0)$ & $2(3)$ & 0.021 \\
\hline Renal failure & $8(5)$ & $5(8)$ & 0.356 \\
\hline Liver failure & $0(0)$ & $1(2)$ & 0.103 \\
\hline Deep venous thrombosis & $2(1)$ & $0(0)$ & 0.385 \\
\hline Ileus & $2(1)$ & $2(3)$ & 0.306 \\
\hline \multicolumn{4}{|l|}{ Minor complications } \\
\hline Wound infection & $9(5)$ & $9(14)$ & 0.025 \\
\hline Wound dehiscence & $3(2)$ & $2(3)$ & 0.522 \\
\hline Urinary tract infection & $0(0)$ & $4(6)$ & 0.001 \\
\hline Reoperation & $17(10)$ & $12(19)$ & 0.064 \\
\hline $\begin{array}{l}\text { Mean time in operating } \\
\text { room }(\mathrm{h})\end{array}$ & 6.1 & 6.3 & 0.384 \\
\hline $\begin{array}{l}\text { Mean length of stay in ICU } \\
\text { (d) }\end{array}$ & 2.5 & 5.0 & $<\mathbf{0 . 0 0 1}$ \\
\hline Mean hospital stay (d) & 21.0 & 27.0 & 0.014 \\
\hline
\end{tabular}

(15 vs. 27\%; $P=0.036$ ), occurred more frequently in group II. Cardiac complications, primarily consisting of arrhythmias, occurred in 27 patients (16\%) versus 24 patients $(38 \%)$ in group II $(P=0.001)$. Pneumonia was the most common infectious complication in 43 patients (18\%), $16 \%$ in group I and $23 \%$ in group II $(P=0.221)$. There were no differences between infectious and noninfectious complications between the two groups $(P=0.5)$. Four abscesses developed in the elderly group (6\%).

The postoperative use of antibiotics and inotropes did not differ statistically between both age groups $(P=0.4$ and $P=0.13$ ).

In logistic regression analysis, age $\geq 70$ years was not a prognostic factor for development of postoperative complications (odds ratio [OR], 1.578; 95\% confidence interval [95\% CI], 0.857 to $2.904 ; P=0.143$ ). For cardiac complications (OR, 3.178; 95\% CI, 1.655 to 6.100; $P=0.001)$ and pulmonary complications (OR, 1.750; $95 \%$ CI, 0.980 to $3.126 ; P=0.05$ ) as a group, age $\geq 70$ years was a prognostic factor (Table 3 ).

There was a higher rate of complications in the patients with comorbidity; 69 (71\%) of the 97 patients who had comorbidity developed one or multiple postoperative complications $(P=0.008)$. However, of the 143 patients who had complications, only 69 (48\%) had preoperative fixed comorbidity. In logistic regression analysis, comorbidity was a prognostic factor for developing postoperative complications (OR, 2.098; 95\% CI, 1.207 to $3.647 ; P=0.009$ ) 
TABLE 3 Prognostic value of advanced age on development of complications in patients with esophageal cancer

\begin{tabular}{|c|c|c|c|}
\hline Variable & Odds ratio & $95 \% \mathrm{CI}$ & $P$ value \\
\hline Overall complications & 1.578 & $0.857-2.904$ & 0.143 \\
\hline Pulmonary complications & 1.750 & $0.980-3.126$ & 0.050 \\
\hline Respiratory insufficiency & 2.085 & $1.135-3.832$ & 0.018 \\
\hline ARDS & 2.041 & $0.444-9.383$ & 0.359 \\
\hline Pneumonia & 1.552 & $0.766-3.146$ & 0.222 \\
\hline Atelectasis & 2.501 & $1.242-5.037$ & 0.010 \\
\hline Pleural effusion & 2.098 & $1.043-4.218$ & 0.038 \\
\hline Empyema & 1.575 & $0.658-3.770$ & 0.308 \\
\hline Pulmonary embolism & 2.041 & $0.444-9.383$ & 0.359 \\
\hline Cardiac complications & 3.178 & $1.655-6.100$ & 0.001 \\
\hline Arrhythmia & 2.971 & $1.542-5.723$ & 0.001 \\
\hline Myocardial infarction & 1.333 & $0.119-14.962$ & 0.816 \\
\hline \multicolumn{4}{|l|}{ Other major complications } \\
\hline Rebleeding & 2.767 & $0.671-11.412$ & 0.159 \\
\hline Subphrenic abscess & & & $\mathrm{np}$ \\
\hline SIRS & 3.711 & $0.807-17.066$ & 0.092 \\
\hline Sepsis & 1.823 & & 0.187 \\
\hline Anastomotic leakage & 0.641 & $0.747-4.449$ & 0.297 \\
\hline Chylothorax & 0.577 & $0.277-1.479$ & 0.490 \\
\hline Intra-abdominal abscess & & & $\mathrm{np}$ \\
\hline Renal failure & 1.716 & $0.121-2.746$ & 0.360 \\
\hline Liver failure & & & $\mathrm{np}$ \\
\hline Deep venous thrombosis & & & $\mathrm{np}$ \\
\hline Ileus & 2.710 & $0.540-5.455$ & 0.324 \\
\hline Minor complications & & $0.374-19.655$ & \\
\hline Wound infection & 2.927 & $1.106-7.748$ & 0.031 \\
\hline Wound dehiscence & 1.796 & $0.293-11.003$ & 0.527 \\
\hline Urinary tract infection & & & np \\
\hline
\end{tabular}

95\% CI, 95\% confidence interval; ARDS, acute respiratory distress syndrome; SIRS, systemic inflammatory response syndrome; np, not enough statistical power

Bold values are significant $(P \leq 0.005)$

\section{Postoperative Course}

The operation time, with a median of 6 hours, was not different in both groups $(95 \% \mathrm{CI},-0.72$ to 0.28 ; $P=0.384)$. The median ICU stay was 3 days, with a range of 1-64 days. In group II, the ICU stay was significantly longer, with a median of 7 days (range, 1-64 days) versus group I, with a median of 3 days (range, 1-56 days) (95\% CI, -9.95 to $-1.86 ; P=0.005$ ). Reoperation was needed in 29 patients as a result of complications, including anastomotic leakage, postoperative bleeding, subphrenic abscess, and obstructive ileus based on torsion at the jejunostomy site. The median hospital stay was 22 days, with 21 days in group I and 26 days in group II (95\% CI, -11.03 to $0.17 ; P=0.06$ ).

\section{Long-Term Outcome}

Median follow-up was 26 months (range, 0 to 199 months), and no patients were lost to follow-up. Follow-up time was not different between the groups $(P=0.701)$.

Stage of disease had no impact on survival between the two groups (stage I, $P=0.298$; stage II; $P=0.834$; stage III; $P=0.184$; stage IVa; $P=2.09$ ).

None of the individual complications had a statistically significant impact on survival. Overall, complications had no influence on long-term survival, including postoperative mortality $(P=0.174)$, and mortality $(P=0.655)$.

Recurrent disease occurred in 126 patients (54\%): 99 patients (58\%) in group I and $27(42 \%)$ in group II $(P=0.028)$. Time to development of recurrent disease did not differ between the groups $(P=0.223)$.

For all 234 patients, including patients with postoperative mortality, the 1 -year survival was $74 \%$ and the 5 -year survival was $33 \%$. There was no difference in survival between the two groups $(P=0.535)$, with a 1 -year survival of $76 \%$ in group I versus $70 \%$ in group II $(P=0.282)$ and a 5 -year survival of $33 \%$ in group I versus $33 \%$ in group II $(P=0.676)$ (Fig. 1$)$.

Age classification of $<70$ and $\geq 70$ years did not have any prognostic value for survival (OR, $1.117 ; 95 \% \mathrm{CI}$, 0.787 to $1.584 ; P=0.535$ ). Also, increasing age as continuous variable did not have a prognostic value for worse survival (OR, $1.005 ; 95 \% \mathrm{CI}, 0.990$ to $1.021 ; P=0.514$ ).

Cancer-related 5-year survival in patients without postoperative mortality $(n=219)$ and cancer-related cause of death was $35 \%$ and did not differ between the groups (35 vs. $37 \%, P=0.874)$.

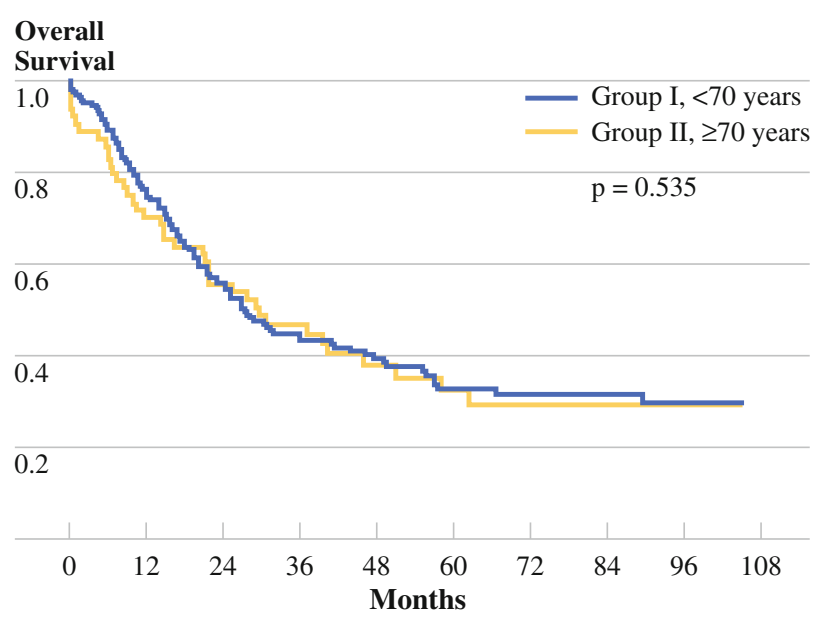

FIG. 1 Kaplan-Meier overall survival curve $(n=234)$ of patients in group I (age $<70$ years) and group II (age $\geq 70$ years) ( $n=170$ vs. $n=64, P=0.535$ ), including postoperative mortality 
Survival curves of comorbidity versus age showed no difference between the two groups $(P=0.135)$. In group I and II separately, there was no statistical difference in survival of patients with and without comorbidity (Fig. 2a, b), although there was a trend for worse survival in elderly patients with comorbidity $(P=0.087)$ (Fig. $2 b)$.

The presence of comorbidity was an independent prognostic factor for long-term survival (OR, 1.679; 95\% CI, 1.219 to $2.314 ; P=0.002$ ). The Kaplan-Meier curve showed significantly better survival for patients without comorbidity in the long term $(P=0.001)$ (Fig. 3).

\section{DISCUSSION}

By applying a thoracotomy-based operative approach with extensive two-field lymph node dissection for esophageal cancer, we were able to effectuate a 5-year cancer-specific survival rate of $35 \%$ in a patient population
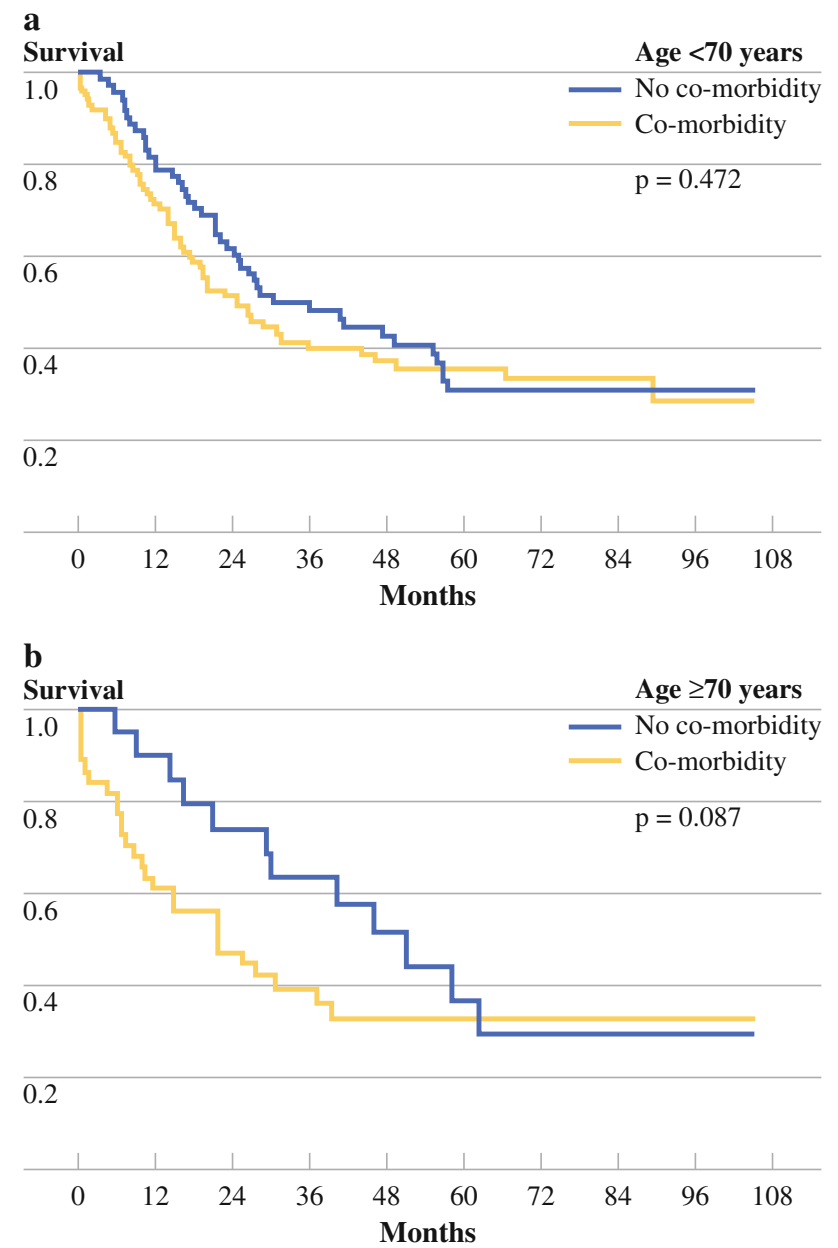

FIG. 2 a Kaplan-Meier survival curve of patients in group I (age $<70$ years) with preoperative comorbidity and those without ( $n=65$ vs. $n=105, P=0.472$ ). b Kaplan-Meier survival curve of patients in group II (age $\geq 70$ years) with preoperative comorbidity and those without ( $n=32$ vs. $n=32, P=0.087$ )

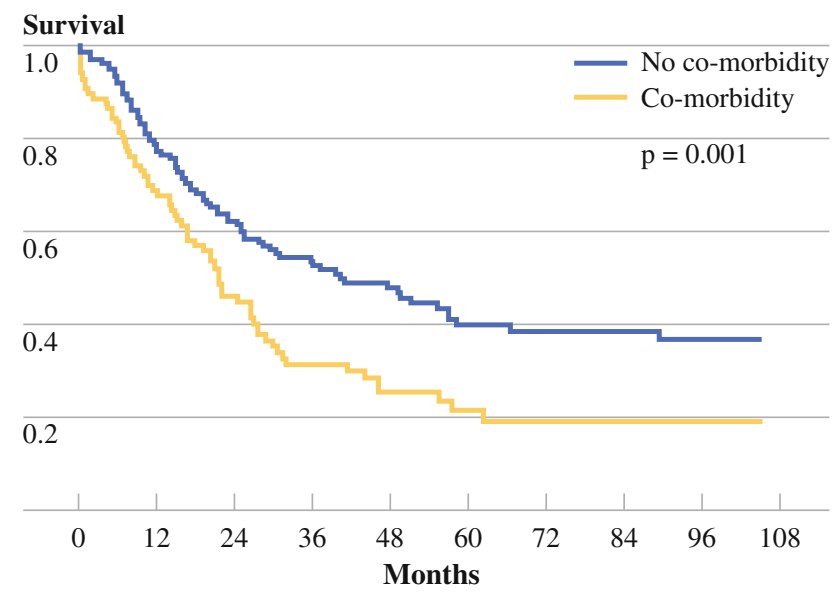

FIG. 3 Kaplan-Meier survival curve of patients with preoperative comorbidity and those without, regardless of age ( $n=97$ vs. $n=137, P=0.001$ )

with $49 \%$ stage III and IV disease, regardless of age and comorbidity.

The median hospital stay was comparable with reported numbers in literature. ${ }^{12}$ Hospital stay is relatively long because some patients with comorbidity in this relatively elderly population needed extensive preoperative workup. Moreover, in our hospital, most patients with anastomotic leakage are usually treated conservatively with good results.

Advanced age ( $\geq 70$ years) had no statistically significant influence on mortality after extended esophagectomy, even though there was a nonstatistical trend of a higher postoperative mortality. Overall, elderly patients had no higher postoperative complication rate than the younger group. However, cardiac complications in particular arrhythmia, and pulmonary complications, especially respiratory insufficiency, atelectasis, and pleural effusion, occurred more frequently in elderly patients. Age $\geq 70$ years was not a prognostic factor for development of postoperative complications. Furthermore, the occurrence of complications did not lead to a decreased survival. On the other hand, comorbidity was the strongest prognostic factor for the development of complications. In this study, cardiovascular comorbidity in the elderly subgroup had a negative effect on postoperative mortality. Because of this relatively small-sized subgroup, it is difficult to give specific recommendations.

Compared with patients $<70$ years old, recurrent disease was statistically significant lower in the elderly group. But the higher number of cardiopulmonary complications and the trend for a higher postoperative mortality in the elderly is concerning.

Along with a general increase of incidence of esophageal adenocarcinoma, there is a rising incidence in esophageal cancer in elderly patients-up to $600 \%$ in the 
last decades. ${ }^{26}$ Advances in treatment of esophageal cancer surgery have been remarkable; improved staging modalities, perioperative management, surgical techniques, and postoperative care have reduced postoperative mortality and morbidity rates and have enhanced better survival. Moreover, improvements in postoperative complications in the elderly have been reported. ${ }^{19}$

Our results reflect the improvement in overall outcomes after esophagectomy for cancer over the last 10 years and demonstrate that this improvement in short-term outcome is evident in the elderly population. ${ }^{20}$ Several studies reported worse postoperative course in the elderly patients, with a high mortality rate and a decreased overall longterm survival with increasing age. ${ }^{20,22}$ More recent studies showed acceptable results regarding mortality and survival because of better surgical techniques, centralization, and more intensive perioperative care. ${ }^{8,10-12,16-19,21,27}$ Therefore, some studies focus on even older patients $(>75$ or $>80$ years). ${ }^{9,12,16}$

Preoperative risk assessment and estimation of prognostic risk factors in the elderly remain controversial. Some studies found a strong association between high age and increased risk of worse prognosis during and after esophagectomy. ${ }^{7,28}$ Particularly cardiac and pulmonary complications occur more frequently in the high age groups. ${ }^{8,16,19,28}$ However, reliable individual risk analysis stratification for individual elderly patients is lacking. This is mainly due to a reluctance to enroll elderly patients in clinical trials, which we think is not appropriate. ${ }^{29}$ This is of importance because the elderly have more cardiopulmonary complications, which complicate the postoperative course. More research is needed for adequate scoring systems identifying the elderly at risk for pulmonary and cardiac complications. ${ }^{8,16,19,28}$ This may permit preoperative intervention such as cardiac and pulmonary support that can reduce the risk of postoperative complications. ${ }^{12}$

In the literature, a discussion is ongoing on the type of surgery required for elderly patients. Some surgeons advocate a limited resection in cases of postoperative complications and coexisting disease in the elderly. However, transthoracic esophagectomy with two-field lymph node dissection is not associated with increased mortality or reduced long-term survival in the elderly population. ${ }^{20}$ In this study, there was a higher mortality rate in the elderly, although it was not statistically significant, but elderly patients had equally long-term survival after surgery. Further optimization in selection criteria and risk stratification for the elderly will better clarify the supposed advantage of extended esophagectomy. Hence, we recommend thorough preoperative assessment in all patients. A threshold to deny surgery on the basis of age alone seems unreasonable in this patient group because of large differences in comorbidity and clinical manifestations of cancer.
A larger study group might strengthen the nonstatistical trends on postoperative mortality in this study, suggesting the need for a large prospective study. The choice to operate on elderly patients with comorbidity remains difficult, but the consequences of not operating may result in an even greater dilemma. The strength of this study is the careful selection of patients for surgery, the homogeneous groups for comparison, and the complete follow-up. It quantifies what the risk is in an experienced center.

More attention is needed in prospective clinical trials for elderly patients, further improving postoperative course and long-term survival. Furthermore, individual risk-analysis stratification should be developed with a focus on patients with comorbidity. Centralization and more intensive perioperative care for elderly patients are needed. Our data support the view that esophageal resection within centralized organized care with a coordinated multidisciplinary approach and multidisciplinary teamwork is feasible and appropriate for all reasonably fit patients, regardless of age. The increased use of neoadjuvant therapy in the elderly patients is needed, especially in clinical trials, with the perception that individualization of treatment will be the future standard. A subdivision based solely on age is undesirable. Elderly patients with no preoperative risk factors may be more readily tolerate chemoradiotherapy and surgery than younger patients with comorbidity.

In conclusion, the increasing life expectancy in the general population will lead to a further increasing incidence of elderly patients with esophageal cancer in the near future. Therefore, more attention is needed for the treatment of the elderly patients. Because this study showed no difference in short- and long-term survival for the elderly group, and elderly patients did not have a substantially worse postoperative course, radical resection should not be withheld in elderly patients. Although age alone is not a prognostic indicator for survival in patients who undergo esophagectomy for cancer, comorbidity at any age might be.

OPEN ACCESS This article is distributed under the terms of the Creative Commons Attribution Noncommercial License which permits any noncommercial use, distribution, and reproduction in any medium, provided the original author(s) and source are credited.

\section{REFERENCES}

1. Enzinger PC. Esophageal cancer. N Engl J Med. 2003;349:224152.

2. Scott Bolton J. Esophagectomy for adenocarcinoma in patients 45 years of age and younger. J Gastrointest Surg. 2001;5:620-5.

3. Stein HJ, Siewert JR. Improved prognosis of resected esophageal cancer. World J Surg. 2004;28:520-5.

4. Pennathur A, Luketich JD. Resection for esophageal cancer: strategies for optimal management. Ann Thorac Surg. 2008;85:S751-6.

5. Sauvanet A, Mariette C, Thomas P, Lozac'h P, Segol P, Tiret E et al. Mortality and morbidity after resection for adenocarcinoma 
of the gastroesophageal junction: predictive factors. $J$ Am Coll Surg. 2005;201:253-62.

6. Bollschweiler E. Preoperative risk analysis in patients with adenocarcinoma or squamous cell carcinoma of the oesophagus. The British journal of surgery 2000;87:1106-10.

7. Abunasra H, Lewis S, Beggs L, Duffy J, Beggs D, Morgan E. Predictors of operative death after oesophagectomy for carcinoma. Br J Surg. 2005;92:1029-33.

8. Ma JY, Wu Z, Wang Y, Zhao YF, Liu LX, Kou YL et al. Clinicopathologic characteristics of esophagectomy for esophageal carcinoma in elderly patients. World $J$ Gastroenterol. 2006;12:1296-9.

9. Moskovitz AH, Rizk NP, Venkatraman E, Bains MS, Flores RM, Park BJ et al. Mortality increases for octogenarians undergoing esophagogastrectomy for esophageal cancer. Ann Thorac Surg. 2006;82:2031-6.

10. Poon RT, Law SY, Chu KM, Branicki FJ, Wong J. Esophagectomy for carcinoma of the esophagus in the elderly: results of current surgical management. Ann Surg. 1998;227:357-64.

11. Sabel MS, Smith JL, Nava HR, Mollen K, Douglass HO, Gibbs JF. Esophageal resection for carcinoma in patients older than 70 years. Ann Surg Oncol. 2002;9:210-4.

12. Internullo E, Moons J, Nafteux P, Coosemans W, Decker G, De LP et al. Outcome after esophagectomy for cancer of the esophagus and GEJ in patients aged over 75 years. Eur J Cardiothorac Surg. 2008;33:1096-104.

13. Peyre CG, Hagen JA, DeMeester SR, Altorki NK, Ancona E, Griffin SM et al. The number of lymph nodes removed predicts survival in esophageal cancer: an international study on the impact of extent of surgical resection. Ann Surg. 2008;248:54956.

14. Lerut T, Nafteux P, Moons J, Coosemans W, Decker G, De Leyn $\mathrm{P}$ et al. Three-field lymphadenectomy for carcinoma of the esophagus and gastroesophageal junction in $174 \mathrm{R} 0$ resections: impact on staging, disease-free survival, and outcome: a plea for adaptation of TNM classification in upper-half esophageal carcinoma. Ann Surg. 2004;240:962-72.

15. Davies AR, Forshaw MJ, Khan AA, Noorani AS, Patel VM, Strauss DC et al. Transhiatal esophagectomy in a high volume institution. World J Surg Oncol. 2008;6:88.

16. Alexiou C, Beggs D, Salama FD, Brackenbury ET, Morgan WE. Surgery for esophageal cancer in elderly patients: the view from Nottingham. J Thorac Cardiovasc Surg. 1998;116:545-53.
17. Ellis FH Jr, Williamson WA, Heatley GJ. Cancer of the esophagus and cardia: does age influence treatment selection and surgical outcomes? J Am Coll Surg. 1998;187:345-51.

18. Jougon JB, Ballester M, Duffy J, Dubrez J, Delaisement C, Velly $\mathrm{JF}$ et al. Esophagectomy for cancer in the patient aged 70 years and older. Ann Thorac Surg. 1997;63:1423-7.

19. Kinugasa S, Tachibana M, Yoshimura H, Dhar DK, Shibakita M, Ohno $\mathrm{S}$ et al. Esophageal resection in elderly esophageal carcinoma patients: improvement in postoperative complications. Ann Thorac Surg. 2001;71:414-8.

20. Rahamim JS, Murphy GJ, Awan Y, Junemann-Ramirez M. The effect of age on the outcome of surgical treatment for carcinoma of the oesophagus and gastric cardia. Eur J Cardiothorac Surg. 2003;23:805-10.

21. Ruol A, Portale G, Zaninotto G, Cagol M, Cavallin F, Castoro C et al. Results of esophagectomy for esophageal cancer in elderly patients: age has little influence on outcome and survival. $J$ Thorac Cardiovasc Surg. 2007;133:1186-92.

22. Thomas P, Doddoli C, Neville P, Pons J, Lienne P, Giudicelli R et al. Esophageal cancer resection in the elderly. Eur J Cardiothorac Surg. 1996;10:941-6.

23. Reid BC, Alberg AJ, Klassen AC, Koch WM, Samet JM. The American Society of Anesthesiologists' class as a comorbidity index in a cohort of head and neck cancer surgical patients. Head Neck. 2001;23:985-94.

24. Sobin LH. TNM, sixth edition: new developments in general concepts and rules. Semin Surg Oncol. 2003;21:19-22.

25. Wittekind C, Compton CC, Greene FL, Sobin LH. TNM residual tumor classification revisited. Cancer. 2002;94:2511-6.

26. Brown LM, Devesa SS, Chow WH. Incidence of adenocarcinoma of the esophagus among white Americans by sex, stage, and age. J Natl Cancer Inst. 2008;100:1184-7.

27. Verhoef C, van de Weyer R, Schaapveld M, Bastiaannet E, Plukker JT. Better survival in patients with esophageal cancer after surgical treatment in university hospitals: a plea for performance by surgical oncologists. Ann Surg Oncol. 2007;14: 1678-87.

28. Ferguson MK, Martin TR, Reeder LB, Olak J. Mortality after esophagectomy: risk factor analysis. World J Surg. 1997;21:599_ 603.

29. Hutchins LF, Unger JM, Crowley JJ, Coltman CA Jr, Albain KS. Underrepresentation of patients 65 years of age or older in cancer-treatment trials. N Engl J Med. 1999;341:2061-7. 